Appl. Set-Valued Anal. Optim. 1 (2019), No. 3, pp. 307-317

Available online at http://asvao.biemdas.com

https://doi.org/10.23952/asvao.1.2019.3.05

\title{
INVERSE PROBLEMS FOR VECTOR VARIATIONAL AND VECTOR QUASI-VARIATIONAL INEQUALITIES
}

\author{
NIKLAS HEBESTREIT ${ }^{1, *}$, AKHTAR A. KHAN ${ }^{2}$, CHRISTIANE TAMMER $^{1}$ \\ ${ }^{1}$ Department of Mathematics, Martin-Luther-University Halle-Wittenberg, Halle (Saale), Germany \\ ${ }^{2}$ Center for Applied and Computational Mathematics, \\ Rochester Institute of Technology, Rochester, New York, USA
}

\begin{abstract}
In this paper, we focus on an inverse problem of parameter identification in vector variational and vector quasi-variational inequalities. Especially, we develop an abstract regularization approach that permits a stable identification of the parameters in the considered variational problems. We give existence results for the regularized output least-square-based optimization problem and provide an application of our results to the Markowitz portfolio problem.
\end{abstract}

Keywords. Inverse problem, vector-variational inequality, vector quasi-variational inequality, regularization.

2010 Mathematics Subject Classification. 49J40, 49J53, 65J20, 65J22.

\section{INTRODUCTION}

Several models in applied sciences lead to variational or quasi-variational inequalities depending on variable coefficients. These coefficients are known, and they characterize properties of the underlying model. The direct problem in this setting is to solve the variational or quasi-variational inequality. In contrast, the inverse problem seeks the coefficient from an estimate of the solution of the associated variational problem, see $[4,9,17]$. Recently, vector variational and vector quasi-variational inequalities appeared as natural and essential generalizations of variational and quasi-variational inequalities, see $[7,12,14,18,20,21,22,24,25]$.

Motivated by the recent research in the fields of inverse problems and vector (quasi) variational problems, in this work, we study the inverse problem of identifying parameters in vector variational and vector quasi-variational inequalities. Vector variational inequalities were introduced in 1980 by Giannessi [8] in a finite-dimensional setting. Since then, several authors have studied and extended this class of problem. Vector quasi-variational inequalities arise from the setting of vector variational problems by attaching a set-valued fixed-point problem to it. In [12], Hebestreit et al. have shown that vector quasi-variational problems can be used to study multi-objective optimization problems with respect to forbidden areas. Compare also [11] for an extensive survey of recent developments in the field of vector variational inequalities and related problems.

\footnotetext{
${ }^{*}$ Corresponding author.

E-mail addresses: niklas.hebestreit@mathematik.uni-halle.de (N. Hebestreit), aaksma@rit.edu (A.A. Khan), christiane.tammer@mathematik.uni-halle.de (C. Tammer).

Received October 14, 2019; Accepted December 21, 2019.
} 
In the following, we describe the general setting of the inverse problem which will be studied in this paper. A similar framework has been used for scalar quasi-variational inequalities in [17]. The parameter space in this work is denoted by $B$ which is assumed to be a Banach space. The set of all admissible coefficients is denoted by $A$. We assume that $A$ is a non-empty, closed and convex subset of $B$. The measured data will be taken in a Banach space $Z$. In order to introduce the vector variational and vector quasi-variational inequality, let $X$ be a reflexive Banach space and $Y$ be a Banach space. The constraining set $C$ is assumed to be a non-empty, closed and convex subset of $X$. In order to compare elements in the abstract space $Y$, we assume that $K$ is a proper, closed, convex, pointed and solid cone in $Y$. Denoting the set of linear and bounded operators from $X$ to $Y$ by $\mathrm{L}(X, Y)$, let $F: B \times X \rightarrow \mathrm{L}(X, Y)$ be a given mapping. The vector variational inequality, depending on the parameter $a \in A$, then reads: given $a \in A$, find an element $x=x(a) \in C$ such that

$$
\langle F(a, x), y-x\rangle_{Y} \notin-\operatorname{int} K, \quad \text { for every } \quad y \in C .
$$

Here, $\langle F(a, x), y-x\rangle_{Y}$ denotes the evaluation of the linear and bounded operator $F x: X \rightarrow Y$ at $y-x$. However, if we let $Y=\mathbb{R}$ and $K=\mathbb{R}_{\geq}$, then $F$ is an operator from $B \times X$ to $\mathrm{L}(X, \mathbb{R})=X^{*}$ and (1.1) recovers the following well-known [15] variational inequality: given $a \in A$, find an element $x=x(a) \in C$ such that

$$
\langle F(a, x), y-x\rangle \geq 0, \quad \text { for every } \quad y \in C .
$$

In the above, $\langle\cdot, \cdot\rangle$ denotes the duality pairing between $X^{*}$ and $X$. In addition to the setting of the vector variational inequality (1.1), let $E: C \rightrightarrows C$ be a given set-valued mapping with non-empty, closed and convex values. By replacing the fixed constraining set $C$ by the set-valued mapping $E$, the vector quasivariational inequality, depending on the parameter $a \in A$, consists of finding an element $x=x(a) \in C$ such that $x \in E(x)$ and

$$
\langle F(a, x), y-x\rangle_{Y} \notin-\operatorname{int} K, \quad \text { for every } \quad y \in E(x) .
$$

Here, the constraining set $E(x)$ depends upon the unknown $x$. In fact, due to this dependence, the solvability of (1.2) is a challenging problem and requires that a vector variational inequality and a setvalued fixed-point problem should be solved simultaneously. Obviously, if the set-valued mapping $E$ is constant, say $E(x)=C$ for every $x \in C$, then (1.2) recovers (1.1) as special case. Clearly, if we let again $Y=\mathbb{R}$ and $K=\mathbb{R}_{\geq}$, then (1.2) recovers the following quasi-variational inequality: given $a \in A$, find an element $x \in C$ such that $x \in E(x)$ and

$$
\langle F(a, x), y-x\rangle \geq 0, \quad \text { for every } \quad y \in E(x) .
$$

The above problem was introduced by Bensoussan and Lions [2] in connection with a problem of impulse control.

Vector variational inequality (1.1) and vector quasi-variational inequality (1.2) constitute the direct problems in this paper. Our main focus however, is on identifying the parameter $a \in A$ from a measurement $z$ of $x(a)$ in these problems. In other words, we seek $a \in A$ such that $x(a)=z$. Since the data might not be regular enough, one can not expect to find such a coefficient and the most commonly adopted approach is to minimize the cost functional

$$
a \mapsto \min _{x \in \mathscr{S}(a)} \frac{1}{2}\|x(a)-z\|_{Z}^{2},
$$

where $\mathscr{S}(a)$ denotes either the solution set of (1.1) or (1.2), depending on the parameter $a \in A$, respectively. Naturally, in order to ensure that the above functional is well-defined, $\mathscr{S}(a)$ must be non-empty for every $a \in A$. However, it is well-known that inverse problems are highly ill-posed in general, that is, they may either have no solution or multiple solutions, and, most importantly, small errors in data could lead to uncontrollable errors in its solution(s). In addition to that, the solution of (1.1) and (1.2) is 
non-unique in general. In order to compensate the ill-posedness of the inverse problem, let $\hat{B}$ be another Banach space, let $R: \hat{B} \rightarrow \mathbb{R}$ be a regularizing mapping and let $\kappa>0$ be a regularization parameter. The key is to chose $\kappa$ small enough so that the regularizer $R$ does not dominate the minimization process, on the other hand large enough to reduce the ill-posed nature of the inverse problem. However, the regularized framework will be specified shortly, see Section 3. In this paper, we study a regularized output-least-square-based optimization problem, which consists of finding a minimizer of the regularized cost functional

$$
a \mapsto J_{\kappa}(a):=\min _{x \in \mathscr{S}(a)} \frac{1}{2}\|x-z\|_{Z}^{2}+\kappa R(a),
$$

where $\kappa>0$ is the regularization parameter and $R$ is the regularizing mapping. The above method is motivated by the work [17]. To the best of our knowledge, this is the first paper where the underlying problem of (1.3) is not scalar but vector-valued.

It is well-known that vector variational problems of the type (1.1) are equivalent to smooth multiobjective optimization problems, see [1]. A famous example is the Markowitz portfolio problem, where the expected return of a portfolio should be maximized while at the same time the risk of the portfolio, measured by the variance, should be minimized. However, if we denote by $\mu$ the expected return vector and by $\Sigma$ the covariance matrix of return, then the Markowitz portfolio problem becomes: given $\mu$ and $\Sigma$, find an element $x=x(\mu, \Sigma) \in C$ such that

$$
\left(\begin{array}{c}
\langle-\mu, y-x\rangle \\
\left\langle\left(\Sigma^{\top}+\Sigma\right) x, y-x\right\rangle
\end{array}\right) \notin-\operatorname{int} \mathbb{R}_{\geq}^{2}, \quad \text { for every } \quad y \in C,
$$

where the constraining set is given by $C=\left\{x \in \mathbb{R}^{l} \mid x_{i} \geq 0\right.$ for $i=1, \ldots, l$ and $\left.\sum_{i=1}^{l} x_{i}=1\right\}$. The above vector variational inequality constitutes the direct problem. If $z$ is a measurement of a solution in an appropriate space, then the inverse problem is to identify the parameters $\mu$ and $\Sigma$ such that $x(\mu, \Sigma)=z$, compare the last section of this paper.

\section{NOTATIONS AND PRELIMINARIES}

In order to make this paper self contained, we briefly set forth below some important notations, definitions and results which we use here.

2.1. Notations. In the following, let $X$ be a Banach space, and let $X^{*}$ be its topological dual. The norm in $X$ and $X^{*}$ will be denoted by $\|\cdot\|_{X}$ and $\|\cdot\|_{X^{*}}$, respectively. The strong convergence, the weak convergence and the duality pairing in any Banach space are specified by the symbols $\rightarrow, \rightarrow$ and $\langle\cdot, \cdot\rangle$, respectively. If $Y$ is another Banach space, we denote by $\mathrm{L}(X, Y)$ the space of all linear and bounded operators from $X$ to $Y$ and $\|\cdot\|_{\mathrm{L}(X, Y)}$ stands for the norm in $\mathrm{L}(X, Y)$. The evaluation of an operator $T \in \mathrm{L}(X, Y)$ at $x \in X$ is denoted by the symbol $\langle T, x\rangle_{Y}$. In the case that $X$ and $Y$ are real finite-dimensional Euclidean spaces, say $X=\mathbb{R}^{l}$ and $Y=\mathbb{R}^{k}$, we use the identification $\mathrm{L}\left(\mathbb{R}^{l}, \mathbb{R}^{k}\right) \cong \operatorname{Mat}_{k \times l}(\mathbb{R})$. Further, with some abuse of the notation, $\langle\cdot, \cdot\rangle$ denotes the Euclidean scalar product.

We further denote the Minkowski sum and difference of two non-empty sets $A, B \subseteq Y$ by $A+B:=$ $\{a+b \mid a \in A, b \in B\}$ and $A-B:=\{a-b \mid a \in A, b \in B\}$. The multiplication of a scalar $\lambda \in \mathbb{R}$ with the non-empty set $A$ is defined by the rule $\lambda A:=\{\lambda a \mid a \in A\}$. In particular, $-A:=(-1) A$. Now, let $K$ be a non-empty subset of $Y$. We call $K$ a cone if $\lambda K \subseteq K$ for every $\lambda \geq 0$. The cone $K$ is called convex if $K+K \subseteq K$, proper if $K \neq\{0\}$ and $K \neq Y$, pointed if $K \cap(-K)=\{0\}$ and solid if the topological interior int $K$ is non-empty. The cone $K$ is said to be closed if $K$ is a closed set in $Y$. See [13,16] for more details. In the Euclidean space $\mathbb{R}^{k}$, the so-called Pareto cone and its interior are given by the formulas $\mathbb{R}_{\geq}^{k}:=\left\{y \in \mathbb{R}^{k} \mid y_{i} \geq 0\right.$ for $\left.i=1, \ldots, k\right\}$ and $\mathbb{R}_{>}^{k}:=\left\{y \in \mathbb{R}^{k} \mid y_{i}>0\right.$ for $\left.i=1, \ldots, k\right\}$. 
2.2. Preliminaries. Let us first recall the following proposition, which will be used frequently, see [10, Lemma 3.9.1] and [26, Proposition 21.23].

Proposition 2.1 (Properties of weak and strong convergence). Let X be a Banach space. We have:

(i) It follows from $x_{n} \rightarrow x$ in $X$ and $f_{n} \rightarrow f$ in $X^{*}$ that $\left\langle f_{n}, x_{n}\right\rangle \rightarrow\langle f, x\rangle$.

(ii) If $X$ is reflexive in addition, then it follows from $x_{n} \rightarrow x$ in $X$ and $f_{n} \rightarrow f$ in $X^{*}$ that $\left\langle f_{n}, x_{n}\right\rangle \rightarrow$ $\langle f, x\rangle$.

(iii) Let $Y$ be another Banach space. It follows from $x_{n} \rightarrow x$ in $X$ and $T_{n} \rightarrow T$ in $\mathrm{L}(X, Y)$ that $\left\langle T_{n}, x_{n}\right\rangle_{Y} \rightarrow\langle T, x\rangle_{Y}$.

We shall now collect some definitions and results for later use.

Definition 2.1 ([5]). Let $X$ and $Y$ be Banach spaces, let $K$ be a proper cone in $Y$ and let $T: X \rightarrow \mathrm{L}(X, Y)$ be a given mapping. The mapping $T$ is called $K$-monotone, if for all $x, y \in X$, we have $\langle T x-T y, x-y\rangle_{Y} \in$ $K$. $T$ is called $v$-hemicontinuous if, for every $x, y, z \in X$, the mapping $\mathbb{R} \rightarrow Y, t \mapsto\langle T(x+t y), z\rangle_{Y}$ is continuous at $0^{+}$.

Remark 2.1. (i) If we let $Y=\mathbb{R}$ and $K=\mathbb{R}_{\geq}$, then the above notions recover the notions of hemicontinuous and monotone operators from $X$ to $X^{*}$, see [27].

(ii) If $T: X \rightarrow \mathrm{L}(X, Y)$ is continuous, then $T$ is $v$-hemicontinuous. Indeed, define $\varphi(t):=\langle T(x+t(y-$ $x)), z\rangle_{Y}$ for $t \in \mathbb{R}$, where $x, y, z \in X$. Then, $\|\varphi(t)-\varphi(0)\|_{Y} \leq\|T(x+t(y-x))-T x\|_{\mathrm{L}(X, Y)}\|z\|_{X}$ and the right-hand side converges to 0 for $t \rightarrow 0^{+}$.

We next recall the vector analogue of the well-known Minty lemma, see [3, 15].

Lemma 2.1. Let $X$ and $Y$ be Banach spaces, $C \subseteq X$ be a non-empty, closed and convex set, and $K$ be a proper, closed, convex, and solid cone in $Y$. Further, let $T: X \rightarrow \mathrm{L}(X, Y)$ be a $K$-monotone and $v$-hemicontinuous mapping. Then, $x \in C$ satisfies

$$
\langle T x, y-x\rangle_{Y} \notin-\operatorname{int} K, \quad \text { for every } \quad y \in C,
$$

if and only if it satisfies

$$
\langle T y, y-x\rangle_{Y} \notin-\operatorname{int} K, \quad \text { for every } \quad y \in C .
$$

The next theorem ensures the existence of solutions of vector variational inequalities. The main tool in the proof of Theorem 2.1 is the famous Fan-KKM lemma. See also Section 5 in [11] for an extensive survey of existence results and solution techniques for vector variational inequalities.

Theorem 2.1 ([3, 25]). Let $X$ and $Y$ be Banach spaces, $C \subseteq X$ be a non-empty, closed and convex set, and $K$ be a proper, closed, convex, and solid cone in $Y$. Further, let $T: X \rightarrow \mathrm{L}(X, Y)$ be a given mapping. Assume that one of the following assumptions is fulfilled:

(i) $T$ is $K$-monotone and $v$-hemicontinuous and the set $C$ is compact.

(ii) $T$ is continuous and the set $C$ is compact.

Then, the following vector variational inequality has a solution: find an element $x \in C$ such that

$$
\langle T x, y-x\rangle_{Y} \notin-\operatorname{int} K, \quad \text { for every } y \in C \text {. }
$$

We further have the following existence result for vector quasi-variational inequalities which is based on a minimal element theorem, compare also [11, Section 11].

Theorem 2.2 ([19]). Let $X$ and $Y$ be Banach spaces, $C \subseteq X$ be a non-empty, closed and convex set, $E: C \rightrightarrows C$ be a set-valued mapping with non-empty, closed and convex values, and $K$ be a proper, closed, convex, and solid cone in $Y$. Further, let $T: X \rightarrow \mathrm{L}(X, Y)$ be a given mapping. Assume that the following assumptions are fulfilled: 
(i) $T$ is continuous.

(ii) The set $C$ is compact in addition.

(iii) The set-valued mapping $E: C \rightrightarrows C$ is upper semicontinuous and has open lower sections.

Then, the following vector quasi-variational inequality has a solution: find an element $x \in C$ such that $x \in E(x)$ and

$$
\langle T x, y-x\rangle_{Y} \notin-\operatorname{int} K, \quad \text { for every } \quad y \in E(x) .
$$

Remark 2.2. Recall that the set-valued mapping $E: C \rightrightarrows C$ is said to have open lower sections if $E^{-1}(x):=\{y \in C \mid x \in E(y)\}$ is open for every $x \in C$. Since $E$ is assumed to have compact values, $E$ is upper semicontinuous if for any sequence $\left\{x_{n}\right\} \subseteq E$ with $x_{n} \rightarrow x$ and for any sequence $y_{n} \in E\left(x_{n}\right)$, there is a subsequence of $\left\{y_{n}\right\}$, again denoted by $\left\{y_{n}\right\}$, such that $y_{n} \rightarrow y$ and $y \in E(x)$.

\section{EXISTENCE RESUlts FOR THE INVERSE Problem}

We now introduce the optimization framework to study the inverse problem of parameter identification in the vector problems. To compensate the ill-posedness of the inverse problem (1.3), we have the following regularized framework:

(R0) The set $A$ of admissible coefficients is a non-empty, closed and convex subset of the Banach space $B$. The observation space $Z$ is a Banach space. $z$ is a given measured data in $Z$.

(R1) $B$ is continuously embedded in a Banach space $L$. There is another Banach space $\hat{B}$ that is compactly embedded in $L$. We further have $A \subseteq B \cap \hat{B}, A$ is closed and bounded in $B$ and also closed in $L . X$ is continuously embedded into $Z$.

(R2) $R: \hat{B} \rightarrow \mathbb{R}$ is a convex and lower-semicontinuous function with respect to $\|\cdot\|_{L}$. Further, for some positive constants $c_{1}$ and $c_{2}$ we have

$$
R(a) \geq c_{1}\|a\|_{\hat{B}}-c_{2}, \quad \text { for every } \quad a \in A .
$$

Recall that, provided the solution set $\mathscr{S}(a)$ is non-empty for every $a \in A$, the regularized output-leastsquare-based optimization problem consists of finding a minimizer of the regularized cost functional

$$
a \mapsto J_{\kappa}(a):=\min _{x \in \mathscr{S}(a)} \frac{1}{2}\|x-z\|_{Z}^{2}+\kappa R(a),
$$

given by (1.3), where $\kappa>0$ is the regularization parameter and $R: \hat{B} \rightarrow \mathbb{R}$ is the regularizing mapping.

3.1. Identification in vector variational inequalities. This section presents two existence results for the identification problem (1.3), where the constraining set $\mathscr{S}(a)$ is given by the solution set of the vector variational inequality (1.1) w.r.t. the parameter $a \in A$. We will use the following assumption:

(A1) $X$ is a reflexive Banach space and $Y$ is a Banach space. $C$ is a non-empty, closed and convex subset of $X . K$ is a proper, closed, convex, and solid cone in $Y$.

We now come to one of the main results of this section.

Theorem 3.1. Besides the assumptions (R0), (R1), (R2) and (A1), let $F: B \times X \rightarrow \mathrm{L}(X, Y)$ be a given mapping such that for every $a \in A, F(a, \cdot): X \rightarrow \mathrm{L}(X, Y)$ is $K$-monotone and v-hemicontinuous. Assume that the following conditions hold:

(i) For every $y \in X$ and every sequence $\left\{a_{n}\right\} \subseteq B$ such that $a_{n} \rightarrow \bar{a}$ w.r.t. $\|\cdot\|_{L}$, it holds that $F\left(a_{n}, y\right) \rightarrow F(\bar{a}, y)$ in $\mathrm{L}(X, Y)$.

(ii) The set $C$ is compact in addition.

Then, identification problem (1.3) for the vector variational inequality (1.1) has a solution. 
Proof. The proof of this theorem is based on Theorem 2.1 and the Minty trick, given in Lemma 2.1. We divide the proof in three parts:

(I). For every $a \in A$, the solution set $\mathscr{S}(a)$ of the vector variational inequality (1.1) w.r.t. the parameter $a \in A$ is non-empty, compare Theorem 2.1 (i). First, let us show that the functional $J_{\kappa}$ given in (1.3) is well-defined. For this, we only need to show that $\min _{x \in \mathscr{S}(a)} I(x)$ is well-defined for $a \in A$, where $I(x):=\frac{1}{2}\|x-z\|_{Z}^{2}$ for every $x \in X$. The functional $I$ is bounded from below since $I \geq 0$. Therefore, there is a minimizing sequence $\left\{x_{n}\right\} \subseteq \mathscr{S}(a)$ such that

$$
\lim _{n \rightarrow+\infty} I\left(x_{n}\right)=\inf _{x \in \mathscr{S}(a)} I(x) .
$$

Consequently, it holds $\left\{x_{n}\right\} \subseteq C$, and due to the compactness of $C$, there is a subsequence which we again denote by $\left\{x_{n}\right\}$ such that $x_{n} \rightarrow \bar{x}$ w.r.t. $\|\cdot\|_{X}$. Obviously, $\bar{x} \in C$. Let us show that $\bar{x} \in \mathscr{S}(a)$. Indeed, since $\left\{x_{n}\right\} \subseteq \mathscr{S}(a)$, we have $x_{n} \in C$ and

$$
\left\langle F\left(a, x_{n}\right), y-x_{n}\right\rangle_{Y} \notin-\operatorname{int} K, \quad \text { for every } \quad y \in C .
$$

Since $F(a, \cdot)$ is $K$-monotone and $v$-hemicontinuous, it holds equivalently

$$
\left\langle F(a, y), y-x_{n}\right\rangle_{Y} \notin-\operatorname{int} K, \quad \text { for every } \quad y \in C,
$$

compare Lemma 2.1. Passing in the above inequality to the limit, compare Proposition 2.1 (iii), and using the fact that the set $Y \backslash(-\operatorname{int} K)$ is closed, we conclude that $\bar{x} \in C$ satisfies

$$
\langle F(a, y), y-\bar{x}\rangle_{Y} \notin-\operatorname{int} K, \quad \text { for every } \quad y \in C .
$$

Consequently, $\bar{x} \in \mathscr{S}(a)$, compare again Lemma 2.1. Since $X$ is continuously embedded into $Z$, it holds $x_{n} \rightarrow \bar{x}$ w.r.t. $\|\cdot\|_{Z}$ in particular. This convergence and the continuity of $I$ yield

$$
\inf _{x \in \mathscr{S}(a)} I(x) \leq I(\bar{x})=\lim _{n \rightarrow+\infty} I\left(x_{n}\right)=\inf _{x \in \mathscr{S}(a)} I(x),
$$

implying

$$
I(\bar{x})=\min _{x \in \mathscr{S}(a)} I(x),
$$

and finally ensuring that $J_{\mathcal{K}}$ is well-defined.

(II). By virtue of the definition of $J_{\kappa}$ given in (1.3) and (R2), we have for every $a \in A$

$$
J_{\kappa}(a)=\min _{x \in \mathscr{S}(a)} I(x)+\kappa R(a) \geq \kappa R(a) \geq \kappa\left(c_{1}\|a\|_{\hat{B}}-c_{2}\right) \geq-\kappa c_{2},
$$

where $c_{1}$ and $c_{2}$ are given by (3.1). In other words, $J_{\kappa}$ is bounded from below. Consequently, there exists a minimizing sequence $\left\{a_{n}\right\} \subseteq A$ for $J_{\kappa}$ such that

$$
\lim _{n \rightarrow+\infty} J_{K}\left(a_{n}\right)=\inf _{b \in A} J_{\mathcal{K}}(b) .
$$

Due to (3.2) and the estimate

$$
\kappa\left(c_{1}\left\|a_{n}\right\|_{\hat{B}}-c_{2}\right) \leq \kappa R\left(a_{n}\right) \leq J_{\kappa}\left(a_{n}\right),
$$

the sequence $\left\{a_{n}\right\}$ is bounded in the Banach space $\hat{B}$. Since $\hat{B}$ is compactly embedded in $L$, there exists a subsequence, again denoted by $\left\{a_{n}\right\}$, that converges in $L$ to an element, say $\bar{a}$. Using (R1), we conclude $\bar{a} \in A$. Subsequently, let $\left\{x_{n}\right\} \subseteq C$ be a sequence such that

$$
x_{n} \in \mathscr{S}\left(a_{n}\right) \quad \text { and } \quad I\left(x_{n}\right)=\min _{x \in \mathscr{S}\left(a_{n}\right)} I(x) .
$$

Since the set $C$ is assumed to be compact, we can find a subsequence of $\left\{x_{n}\right\}$, again denoted by $\left\{x_{n}\right\}$, such that $x_{n} \rightarrow \bar{x}$ w.r.t. $\|\cdot\|_{X}$ and $\bar{x} \in C$. Due to the continuous embedding of $X$ in the observation space 
$Z$, we conclude $x_{n} \rightarrow \bar{x}$ w.r.t. $\|\cdot\|_{Z}$. Let us show that $\bar{x} \in \mathscr{S}(\bar{a})$. Since the mapping $F$ is $K$-monotone and $v$-hemicontinuous in the second argument, it follows similar to the first part of (3.3) that it holds

$$
\left\langle F\left(a_{n}, y\right), y-x_{n}\right\rangle_{Y} \notin-\operatorname{int} K, \quad \text { for every } \quad y \in C,
$$

compare again Lemma 2.1. By assumption (i) and Proposition 2.1 (iii), we have for every $y \in C$

$$
\left\langle F\left(a_{n}, y\right), y-x_{n}\right\rangle_{Y} \rightarrow\langle F(\bar{a}, y), y-\bar{x}\rangle_{Y} \text { in } Y .
$$

Since the set $Y \backslash(-\operatorname{int} K)$ is closed, it follows consequently $\langle F(\bar{a}, y), y-\bar{x}\rangle_{Y} \notin-\operatorname{int} K$. Applying again Lemma 2.1, we conclude that $\bar{x} \in C$ satisfies

$$
\langle F(\bar{a}, \bar{x}), y-\bar{x}\rangle_{Y} \notin-\operatorname{int} K, \quad \text { for every } \quad y \in C .
$$

Thus, $\bar{x} \in \mathscr{S}(\bar{a})$.

(III). Let us finally show that $\bar{a} \in A$ is a solution of (1.3). Since $a_{n} \rightarrow \bar{a}$ w.r.t. $\|\cdot\|_{L}$ and $R$ is assumed to be lower-semicontinuous, it holds

$$
R(\bar{a}) \leq \liminf _{n \rightarrow+\infty} R\left(a_{n}\right) .
$$

Using the relations (3.2), (3.3) and (3.4), we finally deduce

$$
\begin{aligned}
\inf _{b \in A} J_{\mathcal{K}}(b) & \leq J_{\mathcal{K}}(\bar{a})=\min _{x \in \mathscr{S}(\bar{a})} I(x)+\kappa R(\bar{a}) \\
& \leq I(\bar{x})+\kappa R(\bar{a}) \\
& \leq \lim _{n \rightarrow+\infty} I\left(x_{n}\right)+\liminf _{n \rightarrow+\infty} \kappa R\left(a_{n}\right) \\
& \leq \liminf _{n \rightarrow+\infty}\left[I\left(x_{n}\right)+\kappa R\left(a_{n}\right)\right] \\
& =\liminf _{n \rightarrow+\infty}\left[\min _{x \in \mathscr{S}\left(a_{n}\right)} I(x)+\kappa R\left(a_{n}\right)\right] \\
& =\liminf _{n \rightarrow+\infty} J_{\mathcal{K}}\left(a_{n}\right) \\
& =\inf _{b \in A} J_{\mathcal{K}}(b),
\end{aligned}
$$

which implies that $J_{\mathcal{K}}(\bar{a})=\min _{b \in A} J_{\mathcal{K}}(b)$. This shows that $\bar{a}$ is a solution of (1.3). The proof is complete.

Using the idea of the previous proof we have the following result. Here, we replace the $K$-monotonicity assumption of $F$ in the second argument by a continuity condition in both arguments.

Theorem 3.2. Besides the assumptions (R0), (R1), (R2) and (A1), let $F: B \times X \rightarrow \mathrm{L}(X, Y)$ be a given mapping. Assume that the following conditions hold:

(i) $F$ is continuous in the sense that $a_{n} \rightarrow \bar{a}$ w.r.t. $\|\cdot\|_{L}$ and $x_{n} \rightarrow \bar{x}$ w.r.t. $\|\cdot\|_{X}$ implies $F\left(a_{n}, x_{n}\right) \rightarrow$ $F(\bar{a}, \bar{x})$ in $\mathrm{L}(X, Y)$.

(ii) The set $C$ is compact in addition.

Then, optimization problem (1.3) for the vector variational inequality (1.1) has a solution.

Proof. We follow the lines of the previous proof. In order to show that $J_{\kappa}$ given in (1.3) is well-defined, let $a \in A$ and $\left\{x_{n}\right\} \subseteq \mathscr{S}(a)$ be a minimizing sequence for $I$, compare step (I) in the proof of Theorem 3.1. Note that $\mathscr{S}(a)$ is non-empty, see Theorem 2.1 (ii). It remains to show that the limit point $\bar{x}$ of $\left\{x_{n}\right\}$ belongs to $\mathscr{S}(a)$. Indeed, this follows from assumption (i) since

$$
\left\langle F\left(a, x_{n}\right), y-x_{n}\right\rangle_{Y} \rightarrow\langle F(a, \bar{x}), y-\bar{x}\rangle_{Y} \text { in } Y .
$$


Due to the compactness and embedding assumptions, there are sequences $\left\{x_{n}\right\} \subseteq C$ and $\left\{a_{n}\right\} \subseteq A$ such that $x_{n} \in \mathscr{S}\left(a_{n}\right), I\left(x_{n}\right)=\min _{x \in \mathscr{S}\left(a_{n}\right)} I(x), x_{n} \rightarrow \bar{x}$ w.r.t. $\|\cdot\|_{X}$ and $a_{n} \rightarrow \bar{a}$ w.r.t. $\|\cdot\|_{L}$, where $\bar{x} \in C$ and $\bar{a} \in A$. Using assumption (i), it is easily seen that it holds $\bar{x} \in \mathscr{S}(\bar{a})$. Indeed, we have

$$
\left\langle F\left(a_{n}, x_{n}\right), y-x_{n}\right\rangle_{Y} \rightarrow\langle F(\bar{a}, \bar{x}), y-\bar{x}\rangle_{Y} \text { in } Y,
$$

see Proposition 2.1 (iii). Since $C$ and $Y \backslash(-$ int $K)$ are closed sets, we conclude that the limit point $\bar{x} \in C$ satisfies

$$
\langle F(\bar{a}, \bar{x}), y-\bar{x}\rangle_{Y} \notin-\operatorname{int} K, \quad \text { for every } \quad y \in C .
$$

The optimality of $\bar{a}$ for (1.3) follows again from Theorem 3.1, compare step (III). The proof is complete.

3.2. Identification in vector quasi-variational inequalities. The purpose of this section is to present an existence result for the identification problem (1.3), where the constraining set $\mathscr{S}(a), a \in A$, is given by the solution set of the vector quasi-variational inequality (1.2) with respect to the parameter $a$. Similar to the previous section, we need the following assumption:

(A2) $X$ is a reflexive Banach space and $Y$ is a Banach space. $C$ is a non-empty, closed and convex subset of $X . E: C \rightrightarrows C$ is a set-valued mapping with non-empty, closed and convex values. $K$ is a proper, closed, convex, and solid cone in $Y$.

Theorem 3.3. Besides the assumptions (R0), (R1), (R2) and (A2), let $F: B \times X \rightarrow \mathrm{L}(X, Y)$ be a given mapping such that for every $a \in A, F(a, \cdot): X \rightarrow \mathrm{L}(X, Y)$ is $K$-monotone. Assume that the following conditions hold:

(i) $F$ is continuous in the sense that $a_{n} \rightarrow \bar{a}$ w.r.t. $\|\cdot\|_{L}$ and $x_{n} \rightarrow \bar{x}$ w.r.t. $\|\cdot\|_{X}$ implies $F\left(a_{n}, x_{n}\right) \rightarrow$ $F(\bar{a}, \bar{x})$ in $\mathrm{L}(X, Y)$.

(ii) The set $C$ is compact in addition.

(iii) $E: C \rightrightarrows C$ is upper semicontinuous and $E^{-1}(x)$ is open for every $x \in C$. Further, for any sequence $\left\{x_{n}\right\} \subseteq C$ with $x_{n} \rightarrow x$, and for each $z \in E(x)$, there exists $\left\{z_{n}\right\} \subseteq C$ such that $z_{n} \in E\left(x_{n}\right)$ and $z_{n} \rightarrow z$.

Then, the identification problem (1.3) for the vector quasi-variational inequality (1.2) has a solution.

Proof. (I). Let us again show that the functional $J_{K}$ is well-defined. Indeed, Theorem 2.2 implies that the solution set $\mathscr{S}(a)$ is non-empty for every $a \in A$. Let $a \in A$ and denote by $\left\{x_{n}\right\} \subseteq \mathscr{S}(a)$ a minimizing sequence for $I$ such that

$$
\lim _{n \rightarrow+\infty} I\left(x_{n}\right)=\inf _{x \in \mathscr{S}(a)} I(x),
$$

where $I(x):=\frac{1}{2}\|x-z\|_{Z}^{2}$ for every $x \in X$. The compactness of $C$ implies that for an appropriate subsequence it holds $x_{n} \rightarrow \bar{x}$ w.r.t. $\|\cdot\|_{X}$, where $\bar{x} \in C$. We further have $x_{n} \in E\left(x_{n}\right)$ and

$$
\left\langle F\left(a, x_{n}\right), y-x_{n}\right\rangle_{Y} \notin-\text { int } K, \quad \text { for every } y \in E\left(x_{n}\right) .
$$

Let us show that $\bar{x} \in \mathscr{S}(a)$. Due to the upper semicontinuity of $E, \bar{x} \in E(\bar{x})$. Let $z \in E(\bar{x})$ be arbitrarily chosen. By assumption (iii), there is a sequence $\left\{z_{n}\right\} \subseteq C$ such that $z_{n} \in E\left(x_{n}\right)$ and $z_{n} \rightarrow z$ w.r.t. $\|\cdot\|_{X}$. Inserting this sequence into the previous inequality and passing to the limit yields

$$
\left\langle F\left(a, x_{n}\right), z_{n}-x_{n}\right\rangle_{Y} \rightarrow\langle F(a, \bar{x}), z-\bar{x}\rangle_{Y} \text { in } Y,
$$

compare Proposition 2.1 (iii). Since $z \in E(\bar{x})$ was chosen arbitrarily and the set $Y \backslash(-\operatorname{int} K)$ is closed, this implies that $\bar{x} \in E(\bar{x})$ is a solution of the vector quasi-variational inequality (1.2) w.r.t. the parameter 
$a \in A$. In other words, we have shown that $\bar{x} \in \mathscr{S}(a)$. Using similar arguments as in the proof of Theorem 3.1, we conclude that

$$
I(\bar{x})=\min _{x \in \mathscr{S}(a)} I(x)
$$

implying that $J_{\kappa}$ is well-defined.

(II). Let $\left\{a_{n}\right\} \subseteq A$ be a minimizing sequence for $J_{\kappa}$. Again, the sequence is bounded in $\hat{B}$ and consequently $a_{n} \rightarrow \bar{a}$ w.r.t. $\|\cdot\|_{L}$, where $\bar{a} \in A$. Subsequently, denote by $\left\{x_{n}\right\} \subseteq C$ a sequence of solutions such that

$$
x_{n} \in \mathscr{S}\left(a_{n}\right) \quad \text { and } \quad I\left(x_{n}\right)=\min _{x \in \mathscr{S}\left(a_{n}\right)} I(x) .
$$

Using the fact that $C$ is compact, it holds $x_{n} \rightarrow \bar{x}$ w.r.t. $\|\cdot\|_{X}$. Let us show $\bar{x} \in \mathscr{S}(\bar{a})$. Similar arguments as in part (I) of this proof show that $\bar{x} \in \mathscr{S}(\bar{a})$. Indeed, we have again $\bar{x} \in E(\bar{x})$ and for $z \in E(\bar{x})$, we can find a sequence such that $z_{n} \in E\left(x_{n}\right)$ and $z_{n} \rightarrow z$ w.r.t. $\|\cdot\|_{X}$, compare assumption (iii). Consequently,

$$
\left\langle F\left(a_{n}, x_{n}\right), z_{n}-x_{n}\right\rangle_{Y} \rightarrow\langle F(\bar{a}, \bar{x}), z-\bar{x}\rangle_{Y} \text { in } Y .
$$

Since $z \in E(\bar{x})$ was chosen arbitrarily, $\bar{x} \in \mathscr{S}(\bar{a})$.

(III). The optimality of $\bar{a} \in A$ follows similar as in Theorem 3.1. The proof is complete.

\section{ApPlicAtions to The MARKOWITZ PORTFOlio PORTFOlio PROBLEM}

In this section, we are going to consider the famous bicriterial Markowitz portfolio problem, where the expected return of the portfolio should be maximized and the risk of the portfolio, measured by the variance, should be minimized at the same time, see $[6,22,23]$. In other words, the decision maker (investor) wishes to find an optimal portfolio strategy. Since the return of the portfolio is compensated based on the risk of the portfolio, the main task of the investor is to balance the risk-return trade-off for his investments.

To be precise, we consider a market with $l \geq 2$ different financial instruments and returns $r_{i}$ for $i=$ $1, \ldots, l$. We assume that the random variables $r_{i}$ are normal distributed, say $r_{i} \sim \mathscr{N}\left(\mu_{i}, \sigma_{i}^{2}\right)$. The expected value of the random vector $r=\left(r_{1}, \ldots, r_{l}\right)$ is given by $\mu=\left(\mu_{1}, \ldots, \mu_{l}\right)$ and the covariance of the random variables $r_{i}$ and $r_{j}$ is given by $\sigma_{i j}=\mathbb{E}\left[\left(r_{i}-\mu_{i}\right)\left(r_{j}-\mu_{j}\right)\right]$. Further, $\Sigma=\left(\sigma_{i j}\right)_{i j} \in \operatorname{Mat}_{l \times l}(\mathbb{R})$ denotes the covariance matrix of $r$. The Markowitz portfolio problem then reads:

$$
\begin{array}{ll}
\max & \sum_{i=1}^{l} \mu_{i} x_{i} \\
\min & \sum_{i=1}^{l} \sum_{j=1}^{l} \sigma_{i j} x_{i} x_{j} \\
\text { s.t. } & \sum_{i=1}^{l} x_{i}=1 \text { and } x_{i} \geq 0 \text { for } i=1, \ldots, l .
\end{array}
$$

Consequently, introducing the feasible set

$$
C:=\left\{x \in \mathbb{R}^{l} \mid x_{i} \geq 0 \text { for } i=1, \ldots, l \text { and } \sum_{i=1}^{l} x_{i}=1\right\},
$$

we may rewrite (4.1) as the following multi-objective optimization problem: given $\mu$ and $\Sigma$, find an element $x=x(\mu, \Sigma) \in C$ such that

$$
\left(\begin{array}{c}
-\langle\mu, y\rangle \\
y^{\top} \Sigma y
\end{array}\right)-\left(\begin{array}{c}
-\langle\mu, x\rangle \\
x^{\top} \Sigma x
\end{array}\right) \notin-\operatorname{int} \mathbb{R}_{\geq}^{2}, \quad \text { for every } \quad y \in C .
$$


It is easy to check that the components of the objective mapping $\psi((\mu, \Sigma), \cdot): \mathbb{R}^{l} \rightarrow \mathbb{R}^{2}, \psi((\mu, \Sigma), x):=$ $\left(\begin{array}{c}-\langle\mu, x\rangle \\ x^{\top} \Sigma x\end{array}\right)$ for $x \in \mathbb{R}^{l}$, are convex and Gateaux-differentiable. Recall that the covariance matrix $\Sigma$ is symmetric and positive semidefinite. This allows use to rewrite (4.2) as the following [1] vector variational inequality: given $\mu$ and $\Sigma$, find an element $x=x(\mu, \Sigma) \in C$ such that

$$
\langle F((\mu, \Sigma), x), y-x\rangle_{\mathbb{R}^{2}} \notin-\operatorname{int} \mathbb{R}_{\geq}^{2}, \quad \text { for every } \quad y \in C .
$$

Here, the objective mapping $F((\mu, \Sigma), \cdot): \mathbb{R}^{l} \rightarrow \operatorname{Mat}_{2 \times l}(\mathbb{R})$ is defined by

$$
F((\mu, \Sigma), x):=D_{G} \psi((\mu, \Sigma), x)=\left(\begin{array}{c}
-\mu \\
\left(\Sigma^{\top}+\Sigma\right) x
\end{array}\right), \quad \text { for every } \quad x \in \mathbb{R}^{l},
$$

and $D_{G}$ stands for the Gateaux-derivative. Recall that we have $\mathrm{L}\left(\mathbb{R}^{l}, \mathbb{R}^{2}\right) \simeq \mathrm{Mat}_{2 \times l}(\mathbb{R})$. However, since any solution of (4.3) depends on the parameters $\mu \in \mathbb{R}^{l}$ and $\Sigma \in \operatorname{Mat}_{l \times l}(\mathbb{R})$, we are going to study the associated identification problem for the vector variational inequality (4.3), using the framework of Section 3.1. For this purpose, assume that there are numbers $\underline{\alpha}, \bar{\alpha}, \underline{\beta}, \bar{\beta} \in \mathbb{R}$ and $\gamma>0$, given by the decision maker. Let $B:=\mathbb{R}^{l} \times \operatorname{Mat}_{l \times l}(\mathbb{R})$. We introduce the set of admissible parameters by

$$
\begin{aligned}
A:=\left\{(\mu, \Sigma) \in B \mid \underline{\alpha} \leq \mu_{i} \leq \bar{\alpha} \text { for } i=1, \ldots, l,\right. & \\
& \left.\underline{\beta} \leq \Sigma_{i j} \leq \bar{\beta} \text { for } i, j=1, \ldots, l \text { and } x^{\top} \Sigma x \geq \gamma \text { for every } x \in \mathbb{R}^{l}\right\} .
\end{aligned}
$$

The measured data will be taken in $Z:=\mathbb{R}^{l}$. In order to introduce the identification problem, we further let $\hat{B}:=L:=\mathbb{R}^{l} \times \operatorname{Mat}_{l \times l}(\mathbb{R})$. A norm in $\hat{B}$ is given by $\|(\mu, \Sigma)\|_{\hat{B}}=\|\mu\|+\|\Sigma\|$, where $\|\cdot\|$ denotes any norm in $\mathbb{R}^{l}$ and $\operatorname{Mat}_{l \times l}(\mathbb{R})$, respectively. The identification problem then consist of finding a minimizer of

$$
(\mu, \Sigma) \mapsto J_{\kappa}(\mu, \Sigma):=\min _{x \in \mathscr{S}(\mu, \Sigma)} \frac{1}{2}\|x-z\|^{2}+\|\mu\|+\|\Sigma\|,
$$

where $\mathscr{S}(\mu, \Sigma)$ denotes the solution set of (4.3) with respect to the parameter $(\mu, \Sigma) \in A$.

We have the following result.

Theorem 4.1. Using the previous notations, the identification problem (4.4) for the vector variational inequality (4.3) has a solution.

Proof. Let us show that the assumptions of Theorem 3.2 are satisfies in this setting. Indeed, it is easily seen that the abstract framework (R0), (R1) and (R2) holds. In particular, we may define the regularizer $R$ by $R(\mu, \Sigma):=\|\mu\|+\|\Sigma\|$ for $(\mu, \Sigma) \in A$. Obviously, $C$ is a non-empty, convex and compact subset of $\mathbb{R}^{l}$. It remains to show that $F$ is continuous. Indeed, let $\left\{\left(\mu_{n}, \Sigma_{n}\right)\right\}$ and $\left\{x_{n}\right\}$ be sequences such that $\left(\mu_{n}, \Sigma_{n}\right) \rightarrow(\bar{\mu}, \bar{\Sigma})$ w.r.t. $\|\cdot\|_{L}$ and $x_{n} \rightarrow \bar{x}$ w.r.t. $\|\cdot\|$. We consequently have $\mu_{n} \rightarrow \bar{\mu}$ in $\mathbb{R}^{l}$ and $\Sigma_{n} \rightarrow \bar{\Sigma}$ in $\operatorname{Mat}_{l \times l}(\mathbb{R})$, implying $F\left(\left(\mu_{n}, \Sigma_{n}\right), x_{n}\right) \rightarrow F((\bar{\mu}, \bar{\Sigma}), \bar{x})$ in $\operatorname{Mat}_{2 \times l}(\mathbb{R})$. The proof is complete.

\section{CONCLUSiON}

In this paper, we studied inverse problems for vector variational and vector quasi-variational inequalities and gave existence results for the regularized output least-square-based optimization problem. However, it should be noted that our results require the compactness of the constraining set $C$. Therefore, in a forthcoming paper, we wish to replace the compactness assumptions by adequate coercivity conditions.

\section{REFERENCES}

[1] Q. H. Ansari, E. Köbis, J.-C. Yao, Vector Variational Inequalities and Vector Optimization, Springer International Publishing AG, Berlin, 2018.

[2] A. Bensoussan, J. L. Lions, Nouvelle formulation de problemes de controle impulsionenel et applications, C. R. Acad. Sci. Paris 276 (1973), 1189-1192. 
[3] G.-Y. Chen, X.-Q. Yang, The vector complementary problem and its equivalences with the weak minimal element in ordered spaces, J. Math. Anal. Appl. 153 (1990), 136-158.

[4] E. Crossen, M. S. Gockenbach, B. Jadamba, A. A. Khan, B. Winkler, An equation error approach for the elasticity imaging inverse problem for predicting tumor location, Comput. Math. Appl. 67 (2014), 122-135.

[5] G. Chen, X.X. Huang, X. Yang, Vector Optimization: Set-Valued and Variational Analysis, Springer-Verlag, Berlin, Heidelberg, 2005.

[6] M. Ehrgott, K. Klamroth, C. Schwehm, An MCDM approach to portfolio optimization, European J. Oper. Res. 155 (2004), 752-770.

[7] R. Elster, N. Hebestreit, A. A. Khan, C. Tammer, Inverse generalized vector variational inequalities with respect to variable domination structures and applications to vector approximation problems, Appl. Anal. Optim. 3 (2018), 341-372.

[8] F. Giannessi, Theorem of the alternative, quadratic programs, and complementary problems, In: R. W. Cottle, F. Giannessi, J.-L. Lions (eds), Variational Inequalities and Complementarity Problems, John Wiley and Sons, Chichester, 1980.

[9] M. S. Gockenbach, B. Jadamba, A. A. Khan, Equation error approach for elliptic inverse problems with an application to the identification of Lame parameters, Inverse Probl. Sci. Eng. 16 (2008), 349-367.

[10] A. Göpfert, C. Tammer, C. Zălinescu, Variational Methods in Partially Ordered Spaces, Springer-Verlag, New York, 2003.

[11] N. Hebestreit, Vector Variational Inequalities and Related Topics: A Survey of Theory and Applications, submitted 2019.

[12] N. Hebestreit, A. A. Khan, E. Köbis, C. Tammer, Existence theorems and regularization methods for noncoercive vector variational and vector quasi-variational inequalities, J. Nonlinear Convex Anal. 20 (2019), 565-591.

[13] J. Jahn, Vector Optimization, Springer-Verlag, Berlin, Heidelberg, 2011.

[14] D. S. Kim, B. S. Lee, G. M. Lee, N. D. Yen, Vector variational inequality as a tool for studying vector optimization problems, Nonlinear Anal. 34 (1998), 745-765.

[15] D. Kinderlehrer, G. Stampacchia, An Introduction to Variational Inequalities and Their Applications, Academic Press, New York, 1980.

[16] A. A. Khan, C. Tammer, C. Zălinescu, Set-valued Optimization, Springer-Verlag, Berlin, Heidelberg, 2015.

[17] A. A. Khan, D. Motreanu, Inverse problems for quasi-variational inequalities, J. Global Optim. 70 (2018), 401-411.

[18] P. Q. Khanh, L. M. Luu, Some existence results for vector quasivariational inequalities involving multifunctions and applications to traffic equilibrium problems, J. Global Optim. 32 (2005), 551-568.

[19] W. K. Kim, K.-K. Tan, On generalized vector quasi-variational inequalities, Optimization 46 (1999), $185-198$.

[20] I. V. Konnov, J. C. Yao, On the generalized vector variational inequality problem, J. Math. Anal. Appl. 206 (1997), 42-58.

[21] K. L. Lin, D. P. Yang, J. C. Yao, Generalized vector variational inequalities, J. Optim. Theory Appl. 92 (1997), 117-125.

[22] H. Markowitz, Portfolio selection, J. Finance 7 (1952), 77-91.

[23] R. T. Rockafellar, S. Uryasev, Optimization of conditional value-at-risk, J. Risk 2 (2000), 21-41.

[24] F. Usman, S. A. Khan, A generalized mixed vector variational-like inequality problem, Nonlinear Anal. 71 (2009), 5354-5362.

[25] X.-Q. Yang, Vector variational inequality and its duality, Nonlinear Anal. 21 (1993), 869-877.

[26] E. Zeidler, Nonlinear Functional Analysis and its Applications, II/A, Springer-Verlag, New York, Berlin, Heidelberg, 1990.

[27] E. Zeidler, Nonlinear Functional Analysis and its Applications, II/B, Springer-Verlag, New York, Berlin, Heidelberg, 1990. 